\title{
Fetal Medial Habenula Transplants: Innervation of the Rat Interpeduncular Nucleus
}

\author{
Nicholas J. Lenn, P. John Seeley, Pauline M. Field and Geoffrey Raisman \\ Division of Pediatric Neurology, Box 394, \\ University of Virginia Medical Center, Charlottesville, VA 22908 \\ and Laboratory of Neurobiology and Development, \\ National Institute for Medical Research, London, England
}

\section{SUMMARY}

The effects of donor age and site of placement on the survival of fetal medial habenula (MH) transplants into adult rats hosts were examined. The innervation of the interpeduncular nucleus (IPN) in such cases was also examined. Explants of $\mathrm{MH}$ consisting of the medial-dorsal lip of the third ventricle were held in vitro for 1-2 days. Colloidal gold conjugated to wheat germ agglutinin was added for the last 18 hours to label the cells. Four of 16 cases with E19 derived transplants contained donor neurons. Markedly larger transplants were present in $95 \%$ of 20 cases with E16 derived transplants. Sites in the ventral midbrain were successful, while limited or no survival occurred at sites more remote from IPN. Retrograde labeling of transplant neurons was present in each case studied with HRP injection into host IPN. Colloidal gold-labeled macrophages, some oriented capillaries and GFAP-positive processes marked the donor-host interface. In EM the interface was evident only by the difference in tissue elements in the transplant versus host. Numerous synapses of Gray types I and II were present in the transplant. Excellent survival of MH neurons, donor/host interfaces, innervation of IPN by the transplant and fine structure in and around the transplants, all suggest that such preparations are suitable for further experimental analysis of the habenulo-interpeduncular system.

\footnotetext{
Reprints address:

Division of Pediatric Neurology

Box 394, University of Virginia

Medical Center, Charlottesville,

VA 22908, USA
}

\section{INTRODUCTION}

The rat medial habenula (MH) is formed by neurons that undergo their final mitosis on embryonic days (E) $15-18 / 7 /$. Perhaps because of its close proximity to the ventricular zone, $\mathrm{MH}$ is already recognizable at E18, and is well-demarcated at E19. The interpeduncular nucleus (IPN) is the principal site of termination of $\mathrm{MH}$ axons. In the normal adult, the cholinergic endings include conventional en passant synapses in the rostral and central subnuclei and crest synapses in the intermediate subnuclei. The last consist of two cholinergic $\mathrm{MH}$ endings arranged coextensively on either side of a markedly narrowed dendritic sheet, or crest $/ 8 /$. At $90 \%$ of adult crest synapses one of the two endings is from the left $\mathrm{MH}$ and one from the right $/ 6,10,11 /$. The normal development of this highly ordered synaptic organization includes extensive remodeling extending beyond 45 days of age and continued net synaptogenesis until 90 days of age $/ 9 /$. Synaptic plasticity which tends to conserve the normal organization of the cholinergic input is induced by $\mathrm{MH}$ lesions at birth $/ 5 /$ and in the adult $/ 11 /$.

These features of the MH-IPN system support further studies, including afferent target interactions and synaptogenesis in particular. The present study was directed toward explant culture of fetal $\mathrm{MH}$ and subsequent transplantation of $\mathrm{MH}$ into the region of IPN. The goal was to achieve successful transplantation with a period of explant culture to permit future experiments in which a variety of labeling and molecular alterations might be directed analyzing the components of the molecular basis underlying development of IPN's highly-ordered synaptic organization. The principal variables tested were age of donor, site of transplant placement, and methods of analysis. 


\section{MATERIALS AND METHODS}

All host or recipient animals were $180-240 \mathrm{gm}$ females of AS or PVG strains. These are highly inbred strains reared in the germ-free vivarium of the National Institute for Medical Research. Donor fetuses of the same strains were taken by aseptic hysterotomy after purpose-bred, timed pregnancies on embryonic day (E) 16 or 19 (E0 = day of breeding). General anesthesia, induced with $2 \%$ tribromoethanol $1 \mathrm{ml} / 100 \mathrm{gm}$ i.p., was used for hysterotomy, surgery and sacrifice in all cases. Fetuses were decapitated while still anesthetized. Under sterile conditions, the brains were removed from the skull. The diencephalon was exposed dorsally. The entire length of its medial-dorsal lip was removed in one piece by passing a knife along the plane defined by the dorsal and medial grooves which demarcate the habenula at these ages. Explants were incubated in $85 \%$ (w:v) Eagle's minimal essential medium and $15 \%$ heat-inactivated fetal bovine serum supplemented with $15 \mathrm{mM} \mathrm{KCl}, 0.6 \%$ (w:v) glucose, and $2 \mathrm{mM} \mathrm{L}$-glutamine. The outgrowth of processes and centripetal migration of neurons observed when these explants cultures were incubated for 1-2 weeks were evidence of satisfactory culture conditions.

Results from 36 relevant cases are reported here. Donors were E16 (20 cases) or E19 (16 cases). The explants from all of the former and three of the latter were held in vitro for 24 hours, the remainder were in vitro 48 hours. Colloidal gold ( $\mathrm{CAu})$ conjugated to wheat germ agglutinin (WGA) was added $18 \mathrm{hrs}$ prior to transplantation for labeling of donor tissue and transplantation site as previously described /15/. The explants were placed stereotaxically without preceding lesion in 33 cases using a previously described technique /13/. The E19 explants were placed along the course of the fasciculus retroflex in the thalamus, hypothalamus or midbrain tegmentum. The E16 explants were placed in the midline in the mammillary body, between the fasciculus retroflexus of each side in the roof of the interpeduncular fossa or in the ventral midbrain adjacent to the IPN. In 3 cases, E19 explants were placed into aspiration cavities which ablated $\mathrm{MH}$ and followed the fasciculus retroflexus from $\mathrm{MH}$ to its midcourse in the midbrain tegmentum.

Host animals were sacrificed by perfusion 14 to 58 days after transplantation. For microscopic preparation, $10 \%$ formalin, or $1 \%$ paraformaldehyde and $1.25 \%$ glutaraldehyde in $0.1 \mathrm{M}$ phosphate buffer, were used. For transport and other slice experiments, freehand slices of midbrain were rapidly cut after perfusion of $32^{\circ} \mathrm{C}$ culture medium gassed with $95 \% \mathrm{O}_{2}, 5 \%$ $\mathrm{CO}_{2}$. The slices were in a bath of the same medium within 10 minutes of the start of surgery. One hr later, they were injected with HRP (Boehringer-Mannhein) or CAu conjugated to FITC-WGA (Miles Laboratories) as above which were dried onto the tip of a micropipet. After $4 \mathrm{hrs}$ further incubation, they were immersed in one of the above fixatives. Slices and blocks were sectioned with a vibratome or after embedding in paraffin or Durcupan. Selected sections were reacted with diaminobenzidine for HRP, silver-enhanced to visualize the $\mathrm{CAu} / 2 /$, stained with thionin, toluidine blue (1 um plastic sections) and for EM /9/, or immunostained for glial fibrillary acidic protein or neurofilament protein with the PAP method.

\section{RESULTS}

Four cases with E19 explants, when examined 17-58 days later, contained small transplants marked with colloidal gold. In these cases and the more successful E16 cases (see below), the colloidal gold provided an unambiguous marker for the transplants. These transplants contained small numbers of neurons consistent in appearance with in situ $\mathrm{MH}$ neurons. There were variable numbers of macrophages and other unidentified cells containing colloidal gold in these animals and in three others. The site of placement varied from the upper half of the posterior diencephalon to the ventral surface of the posterior diencephalon in or near the mammillary body. Two of the four cases had placement nearer to IPN. The remaining 11 cases, including those with colloidal gold present in macrophages and other cells of probable host origin within the transplant cavity, contained no donor neurons and are considered negative. The negative cases as a group had longer survival times. Since animals sacrificed within 72 hours of transplantation (5 cases not otherwise reported) were observed microscopically to have transplants present, there was presumably loss of transplanted tissue by atrophy or necrosis from these E19 cases over time.

When E16 MH were used, transplants were present in 19 of 20 cases examined 17-42 days later (Figs. 1-4). They were large (up to $1.5 \mathrm{~mm} \times 3 \mathrm{~mm}$ ) in 12 cases. All of these transplants were in the roof of the interpeduncular fossa, rostrodorsal or lateral to IPN, extending in some cases into the mammillary body. Colloidal gold was present in each case as seen with $\mathrm{Ag}$ enhancement or EM. It was predominantly in lysosomes of macrophages, astrocytes and pericytes. 
Such macrophages were concentrated near the border of the transplants, possibly because of limited penetration of $\mathrm{CAu}$ into the explants. The border of the transplants was also accentuated by an accumulation of GFAP-immunostained processes which appeared limited to the host side. There were faintly GFAPpositive processes scattered within the transplants, but in contrast to the positive processes on the host side, those within the transplants showed no accumulation in the region of the host-donor interface. The expected, normal-appearing GFAP-positive cell bodies and processes were present in the remainder of the midbrain. In the $1 \mathrm{um}$ and EM sections, the border was noticeable because the transplants had a tissue pattern similar to normal $\mathrm{MH}$, contrasting to the structure of the respective host areas across distinct interfaces (Fig. 1). In semi-thin plastic sections, the donor neurons had features, including central, vesicular nuclei, large central nucleoli, and circular perikarya, which are characteristic of, but not unique to, $\mathrm{MH}$ neurons (Figs. 1-2). Furthermore, many of these perikarya had extensive lengths of direct membrane-to-membrane contact with one another, which is also characteristic of MH. Serial $1 \mathrm{um}$ sections from normal rats through the regions of these transplant sites were examined to confirm that there is no normal group of neurons in this region with these features.

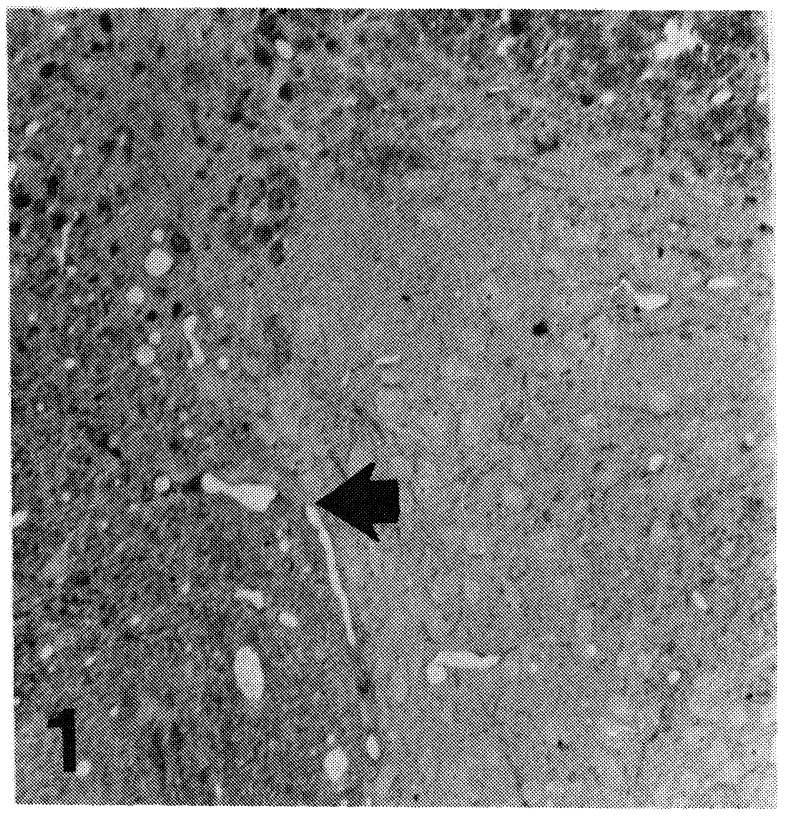

Fig. 1. Transplant (right) and part of the host interpeduncular nucleus (left). The intimate smoothly curved border between these two structures (arrow) is highlighted by the contrast of the closely-spaced vesicular neurons in the transplant to the small myelinated fibers in this lateral part of IPN. $\times 114$.

Figs. 1-3. Light and electron micrographs of E16 fetal medial habenula transplanted into adult ventral midbrain. Perfusion fixation $\mathbf{3 2}$ days later.

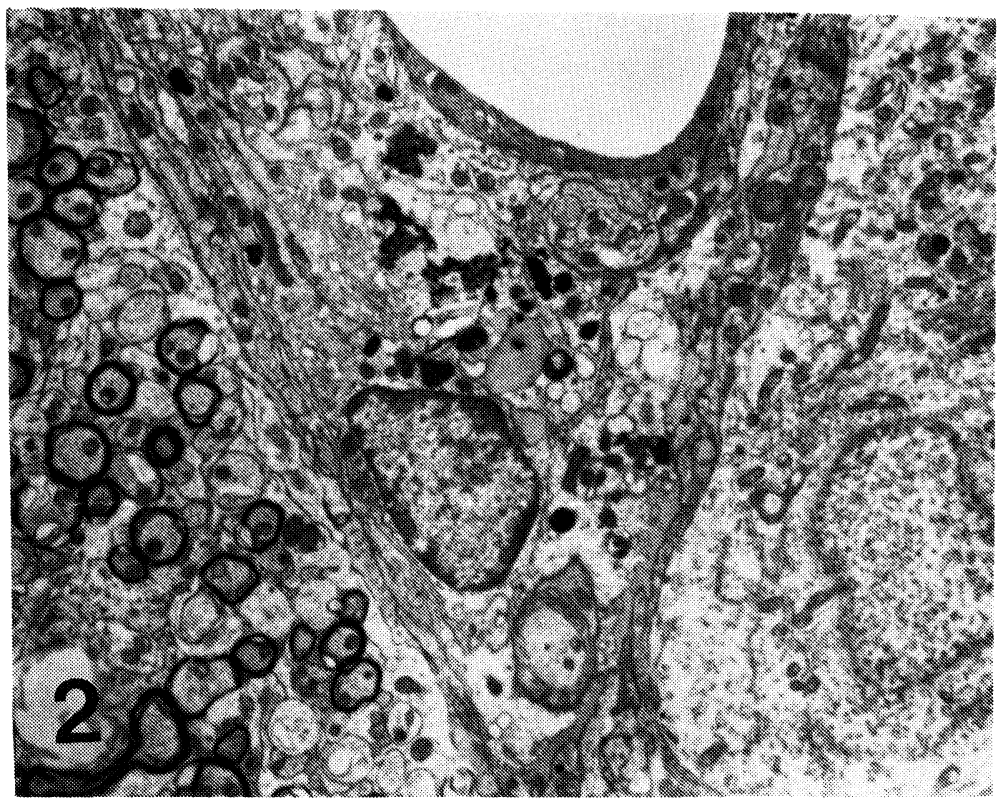

Fig. 2. Electron micrograph showing the border between host (left) and transplant (right). The capillary and perivascular cells separate a neuron within the transplant and the myelinated axons in lateral IPN. The lysosomes in the pericytes can be seen to contain colloidal gold at higher resolution (not shown). $\times 4,200$. 


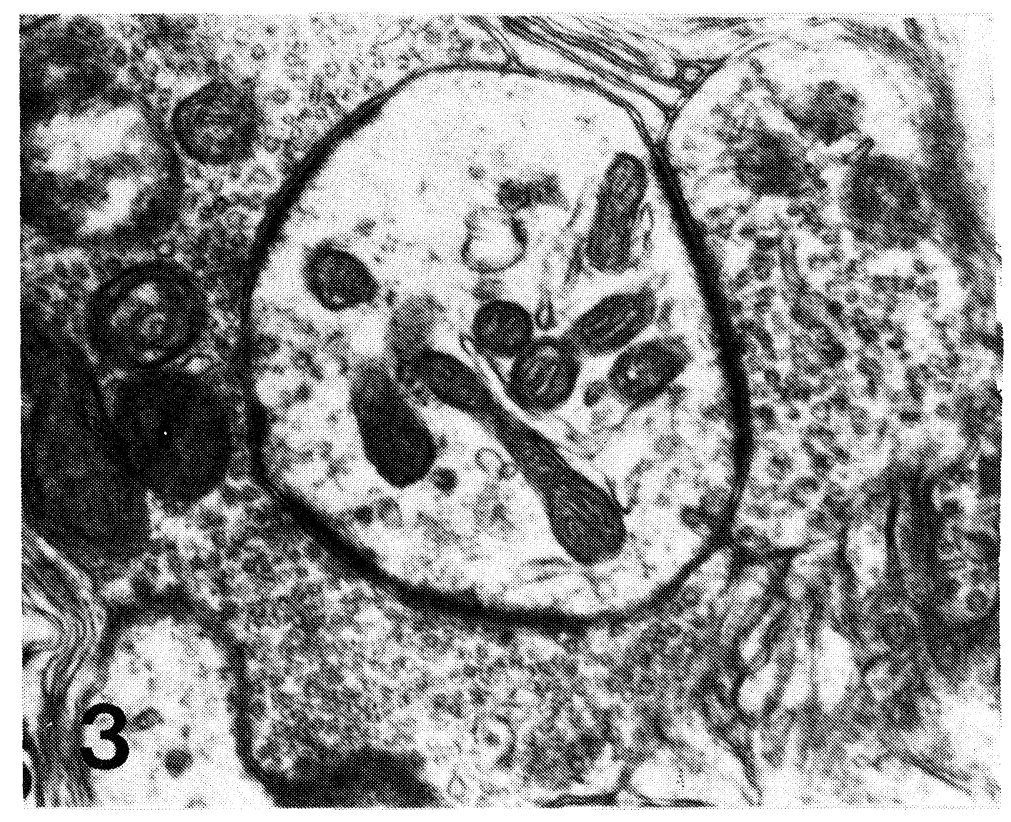

Fig. 3. Electron micrograph showing examples of the apparently exodendritic synapses frequently encountered throughout the transplant. These are probably intrinsic connections. $\times 28,000$.

Throughout the sections examined, virtually all surviving neurons seen in these transplants were $\mathrm{MH}$ cells. This is in spite of the fact that the explants had originally contained lateral habenula, ventricular zone, ependyma and probably small portions of nearby thalamic nuclei.

The fine structure of the transplants was remarkably normal. The EM again showed the features of the transplant borders seen in the light micrographs (Fig. 2). No differences were appreciated between the transplants and normal MH from control animals (Lenn, unpublished observations). Numerous synapses were present, with a variety of presynaptic terminals making Gray type I or II contacts with moderate size dendritic processes (Fig. 3). No unusual pre- or postsynaptic features were present. Under the circumstances these synapses are presumed to be connections between donor neurons. The capillaries were of normal structure for brain, with gap junctions, basal lamina and occasional pericytes, but without fenestrations. There were no abnormal spaces, extravasated red blood cells or other discontinuities, even at the borders. The fine structure of the host IPN was normal. However, since the relevant experiments were done without MH lesions, the synapses present are presumed to be predominantly of the host origin. There was colloidal gold present in occasional astrocytic processes in portions of IPN near to and remote from the transplant. None was found in axons or their terminals.
Three cases were prepared as slices and injected with HRP into IPN during in vitro incubation. The injection sites conformed to the entire IPN with slight spread into the superior cerebellar peduncule dorsally and medial lemniscus laterally. Some HRP staining of the surface of the slices was present due to a very low concentration of HRP in the bath from leakage during incubation. However, this surface reaction was light, diffuse, and limited to a small fraction of the height of the transplants. There was an HRP-free region anterior to IPN in each case separating the transplant from the injection site. The transplants in each of these cases were $1 \mathrm{~mm} \times 2-3 \mathrm{~mm}$. Each was located in the midline, rostral to IPN and caudal to the mammillary body. The retrograde labeling produced diffuse filling of the perikarya, proximal dendrites and axons of numerous neuronal perikarya within the transplants (Fig. 4). The different appearance of $\mathrm{MH}$ neurons between this material and the EM specimens, including semi-thin plastic sections, is attributable to the much greater thickness of the sections and the appearance of the dendrites and axons extending from the cell bodies.

\section{DISCUSSION}

The present study demonstrates several features of neural transplantation of $\mathrm{MH}$. Of the several variables tested, it is quite clear that the gestational age of the 


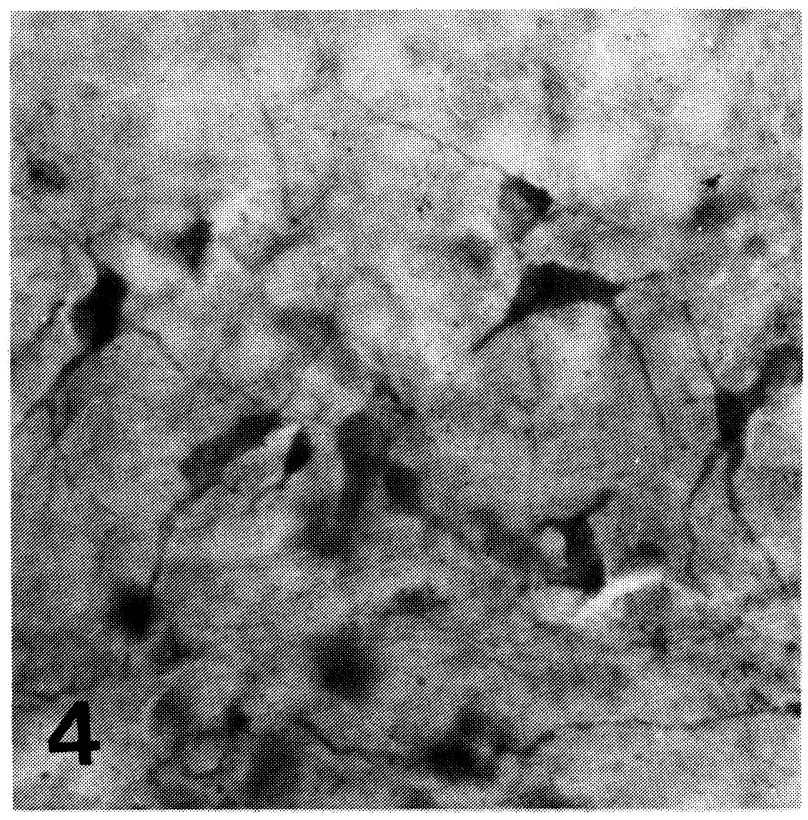

Fig. 4. Light micrograph of an E16 MH transplant into ventral midbrain rostral to IPN showing retrograde HRP-filling of transplant neurons, including perikarya, proximal dendrites and axons, following in vitro injection of HRP into host IPN 17 days after implantation. $\times 250$.

donor tissue can account for most or all of the striking difference in survival and growth of the MH transplants. This is in agreement with previous evidence that the ontogenetic stage of the donor tissue is important in these regards $/ 1,3,16 /$. It appears that the optimal age may approximate the time of the final mitoses of the neurons to be transplanted. This does not exclude a role for the ventricular zone itself if included in the transplant, as was the case here, in that the surviving neurons might have been generated in part by mitoses which occurred after transplantation. It does suggest that transplantation prior to or early in the time when neurons would normally be extending processes and prior to when they would contact their target is important for survival and innervation of the host. This suggestion is also supported by the convincing, preferential survival of $\mathrm{MH}$ neurons in the present transplants. The original explants (and identical explants when fixed and stained before or on the first day after transplantation) contained lateral habenula (LH) and the portion of ventricular zone from which its neurons arise $/ 7 /$. However, LH and $\mathrm{MH}$ cells are at different points in their life cycle at explant, a potential factor in their differential survival.
A second factor probably contributing to the preferential survival of $\mathrm{MH}$ cells is their success in contacting their normal target, and the failure of LH neurons to contact a suitable target $/ 12 /$. The presence of several normal lateral habenular targets in the vicinity of these transplants militates against the possibility that the sites of transplantation chosen favored MH. Dependence on target contact, if correct, may imply a requirement for a trophic substance on which these immature neurons are dependent, and whose source is at their target area. Furthermore, there might be a critical period in the life cycle of the donor neurons, such that with E19 donors one or more of these hypothetical steps no longer occurs successfully. However, caution in accepting such an interpretation is necessary pending direct experimental evidence. Transplantation experiments involving other regions have added new exceptions to the generalization that neurons must contact their normal target to survive $/ 4$, $18,20 \%$. Furthermore, there is evidence that the age of the host animal is important in the survival and/or innervation by transplants $/ 3,18 /$, and for an effect of deafferenting lesions on the latter $/ 14,17,19 /$. The excellent survival of $\mathrm{MH}$ neurons, donor/host interfaces, innervation of IPN by the transplant and fine structure in and around the transplants observed in the present experiments, all indicate that transplantation will be useful for studying the development of IPN.

\section{ACKNOWLEDGEMENTS}

Supported in part by a Fogarty Senior International Fellowship (FO6 TW01294) and NIH grant \#NS 16882, both to N.J.L. The authors thank Ms. Ursula Starega and Leanna Whitmore for technical assistance, and Dr. Scott Vandenberg for assistance with immunostains.

\section{REFERENCES}

1. Cree BC, Smith LM and Ebner FF. The synaptic composition of neocortical transplants, a quantitative analysis, Neurosci. Abst., 13, 1458 (1987).

2. Danscher G. Light and electron microscopic localization of silver in biological tissue. Histochemistry, 71, 177-186 (1981).

3. Das GD. Neural transplantation in mammalian brain: some conceptual and technical considerations. In R.B. Wallace and G.D. Das (Eds.), Neural Tissue Transplantation Research. Springer-Verlag, New York Berlin Heidelberg Tokyo, pp. 1-64 (1983). 
4. Gibbs RB, Anderson $\mathrm{K}$ and Cotman $\mathrm{CW}$. Factors affecting innervation in the CNS: comparison of three cholinergic cell types transplanted to the hippocampus of adult rats. Brain Res., 383, 362-366 (1986).

5. Hamill GS and Lenn NJ. Synaptic plasticity within the interpeduncular nucleus after unilateral lesions of the habenula in neonatal rats. J. Neurosci., 3, 2128-2145 (1983).

6. Lenn NJ. Synapses in the rat interpeduncular nucleus: an electron microscopic study. J. Comp. Neurol., 176, 73-100 (1976).

7. Lenn NJ and Bayer SA. Neurogenesis in subnuclei of the rat interpeduncular nucleus and medial habenula. Brain Res. Bull., 16, 219-224 (1986).

8. Lenn NJ, Leranth Cs and Zaborszky L. Choline acetyltransferase immunoreactivity is localized to four types of synapses in the rat interpeduncular nucleus. J. Neurocytol., 14, 909-919 (1985).

9. Lenn NJ and Whitmore L. Modification of left-right pairing during the development of individual crest synapses in the rat interpeduncular nucleus. J. Comp. Neurol., 281, 136-142 (1989).

10. Lenn NJ, Wong $\mathrm{V}$ and Hamill GS. Left-right pairing of afferents at the crest synapses of rat interpeduncular nucleus. Neuroscience, 9, 383-389 (1983).

11. Murray M, Raisman G and Zimmer J. Reinnervation in the rat interpeduncular nucleus after lesions of the fasciculus retroflexus. J. Comp. Neurol., 186, 34-51 (1979).

12. Oppenheim RW. Muscle activity and motor neuron death in the spinal cord of the chick embryo. In Selective Neuronal Death, Ciba Fn. Symp., Vol. 126, Wiley, Chichester, pp. 96-112 (1987).
13. Raisman G and Ebner FF. Mossy fibre projections into and out of hippocampal transplants. Neuroscience, 9, 783-801 (1983).

14. Raisman G, Morris RJ and Zhou C-F. Specificity in the reinnervation of adult hippocampus by embryonic hippocampal transplants. In F.J. Seil, Herbert E. and Carlson B.M. (Eds.), Progress in Brain Research. Vol. 71, Elsevier Sci. Publ., Amsterdam, pp. 325-333 (1987).

15. Seeley PJ and Field PM. Use of colloidal gold complexes of wheat germ agglutinin as a label for neural cells. Brain Res., 449, 177-191 (1988)

16. Stenevi U, Bjorklund A and Svendgaard N-A. Transplantation of central and peripheral monoamine neurons in the adult rat brain: techniques and conditions for survival. Brain Res., 114, 1-20 (1976).

17. Sunde NA and Zimmer J. Transplantation of central nervous tissue. An introduction with results and implications. Acta Neurol. Scandinav., 63, 323-335 (1981).

18. Sunde NA and Zimmer J. Cellular, histochemical and connective organization of the hippocampus and fascia dentata transplanted to different regions of immature and adult rat brains. Dev. Brain Res., 8, 165-191 (1983).

19. Toniolo G, Dunnett SB, Hefti F and Will B. Acetylcholinerich transplants in the hippocampus: influence of intrinsic growth factors and application of nerve growth factor on choline acetyltransferase activity. Brain Res., 345 , 141-146 (1985).

20. Wiegand SJ and Gash DM. Organization and efferent connections of transplanted suprachiasmatic nuclei. J Comp. Neurol., 267, 562-579 (1988). 

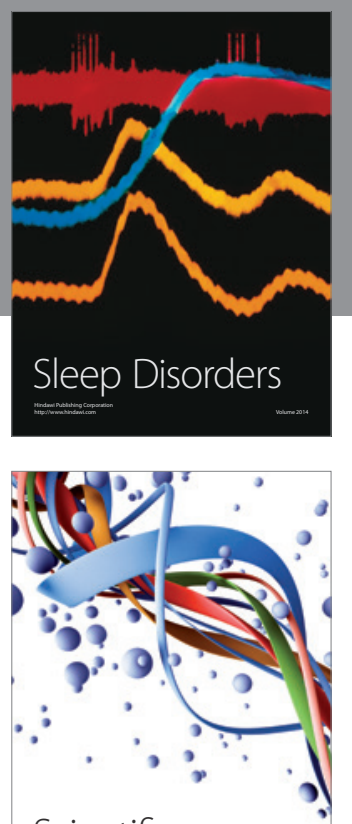

Scientifica
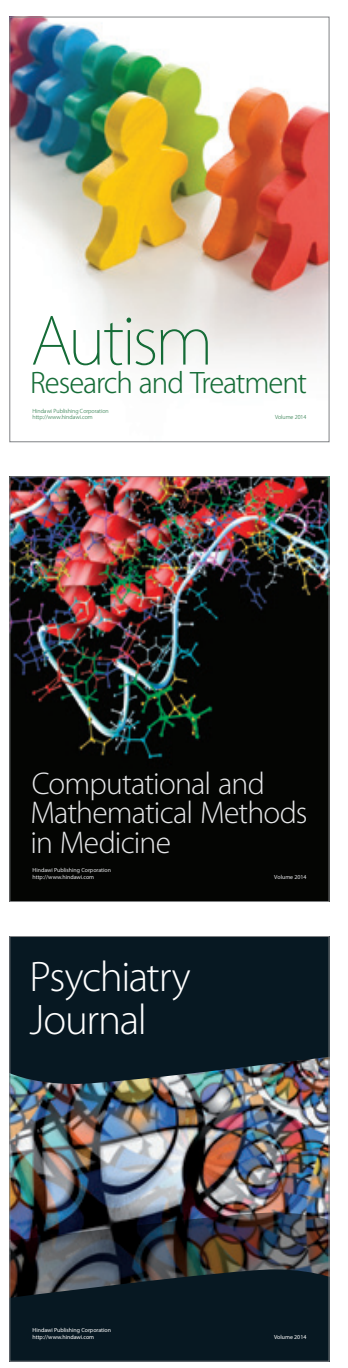
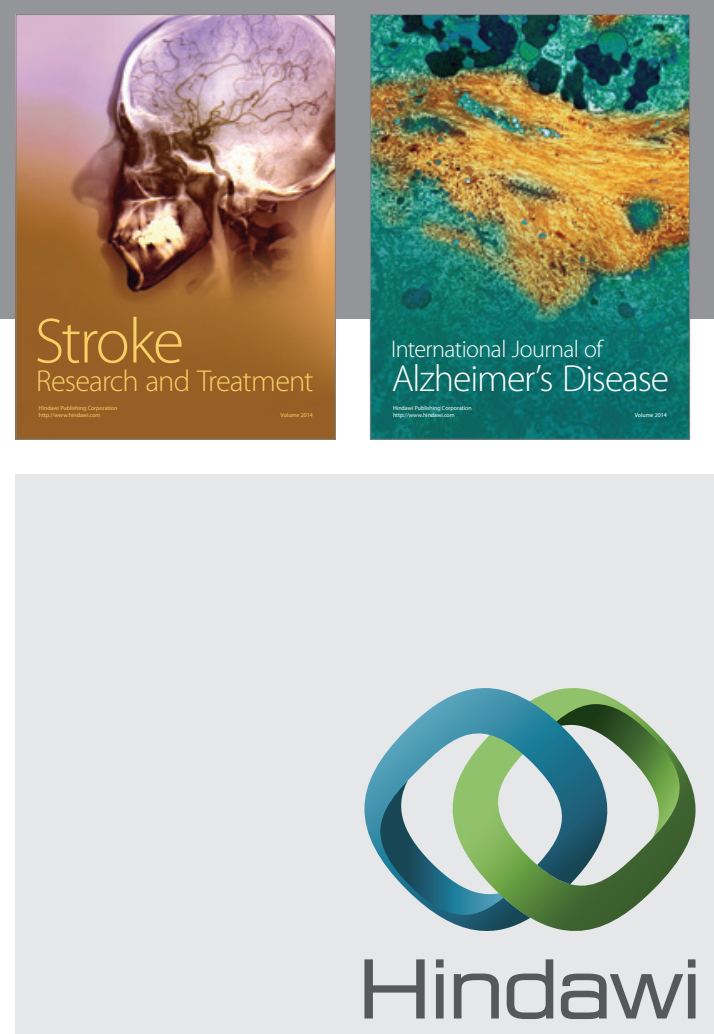

Submit your manuscripts at

http://www.hindawi.com
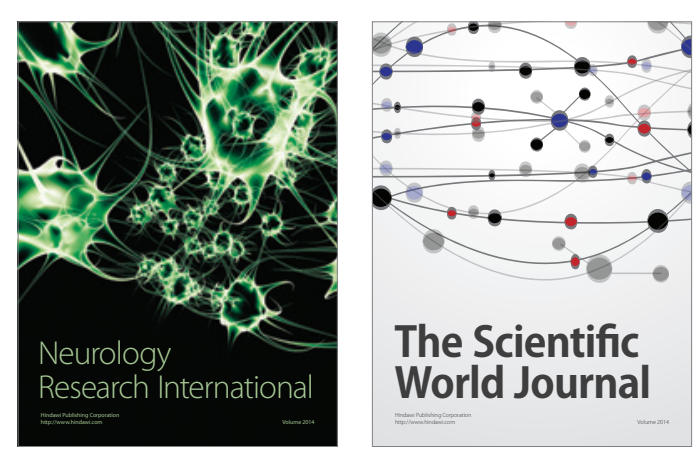

The Scientific World Journal

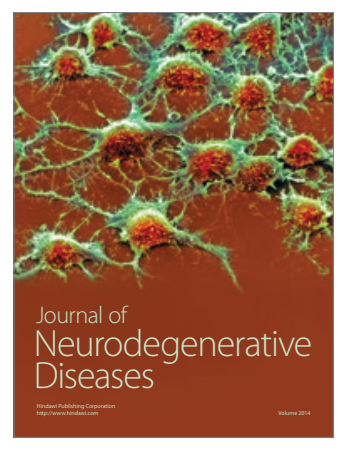

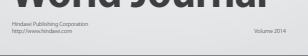

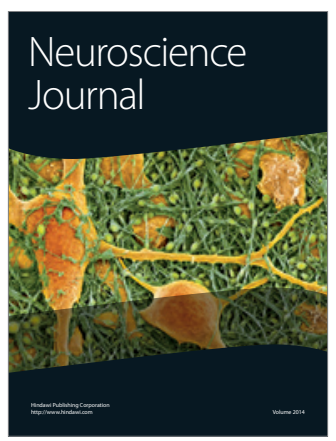

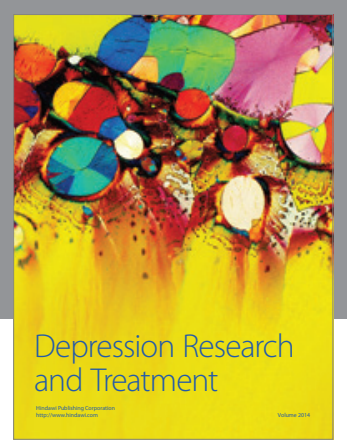
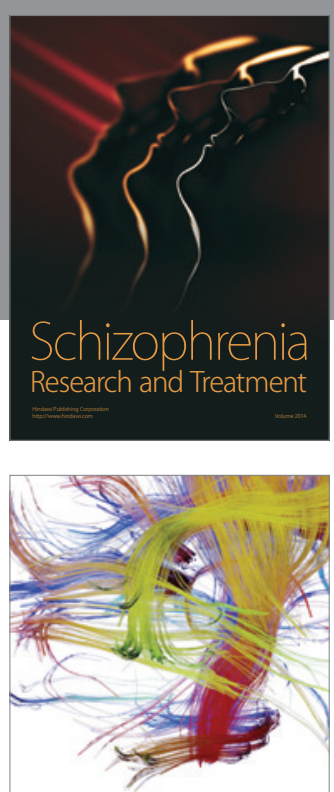

Brain Science

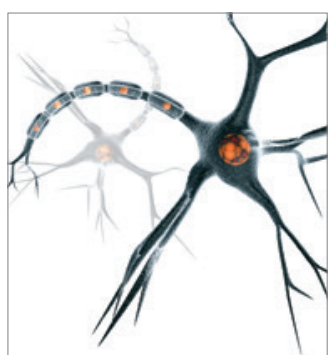

Neural Plasticity
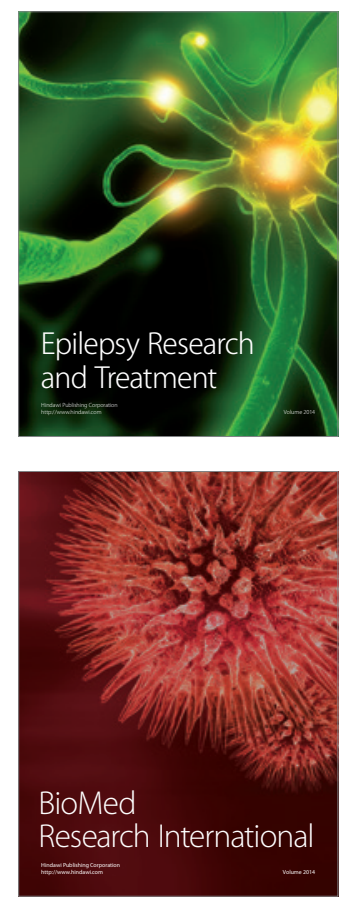

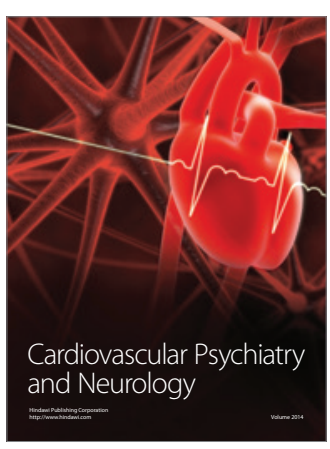

Parkinson's

Disease
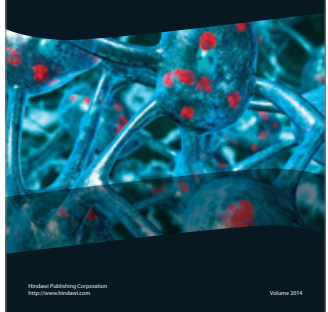